Mathematical Research Letters 7, 537-549 (2000)

\title{
FONCTIONS HOLOMORPHES SUR L'ESPACE DES CYCLES: LA METHODE D'INTERSECTION
}

\author{
D. BARlet et V. KoziARZ
}

\section{Introduction}

Si $X$ est une variété complexe de dimension $N$, il y a deux méthodes "classiques" pour construire des fonctions holomorphes sur $\mathcal{C}_{q}(X)$, l'espace des $q$ cycles compacts de $X$.

La première, due à A. Andreotti et F. Norguet, consiste à intégrer des $(q, q)$ classes de cohomologie sur les $q$-cycles; elle a été introduite dans [AN67]. C'est une méthode "cohomologique" et elle a l'avantage de produire beaucoup de telles fonctions en présence d'une hypothèse de $q$-convexité forte (ici 0-complet=Stein) grâce au théorème de finitude d'Andreotti-Grauert. La seconde méthode, initiée dans [Ba78] est indirecte, puisqu'elle consiste essentiellement à déduire d'une exhaustion $q$-convexe sur $X$ une exhaustion 0-convexe $\operatorname{sur} \mathcal{C}_{q}(X)$. La réciproque du problème de Levi donnant alors l'existence des fonctions holomorphes voulues.

Nous nous proposons d'introduire ici une troisième méthode, plus géométrique, consistant à couper les $q$-cycles par un sous-ensemble analytique fermé $Y$ de codimension $q$ sur lequel il y a beaucoup de fonctions holomorphes (par exemple, un sous-ensemble fermé de Stein). La trace d'une telle fonction holomorphe calculée sur l'intersection (finie) du $q$-cycle $\gamma$ avec $Y$ donnera la valeur en $\gamma$ de la fonction holomorphe désirée.

En fait, la considération de la classe fondamentale de $Y$ permet assez facilement de voir que l'on construit ainsi des $(q, q)$-classes de cohomologie que l'on intègre sur les $q$-cycles. On est donc plus ou moins ramené à la première méthode. Tout l'intérêt réside en fait dans l'origine géométrique des $(q, q)$-classes de cohomologie qui ont un caractère plus explicite et concret que les classes que l'on peut construire grâce à un théorème de finitude de la cohomologie. Nous obtenons d'ailleurs quelques résultats qui semblent échapper aux précédentes méthodes. Il est également intéressant de constater qu'une adaptation locale de cette méthode permet de donner une propriété de 0 -convexité locale en un point du bord (correspondant à un cycle $\gamma$ ) d'un ouvert de la forme $\mathcal{C}_{q}(U)$, pour $U$ ouvert relativement compact avec une propriété de $q$-convexité locale près des points du bord

Received February 16, 2000.

2000 Mathematics Subject Classification. 32E99, 32F10, 14C17

Key words and phrases. Espaces de cycles, convexité holomorphe relative, convexité locale, intersection de cycles 
de $U$ qui sont dans le cycle $\gamma$. Ceci est en fait une version géométrique locale de [AN66].

En général, le support d'un cycle $\gamma$ est noté $|\gamma|$, mais il pourra nous arriver de le noter simplement $\gamma$, lorsqu'il n'y aura pas de confusion possible.

\section{Holomorphie des traces sur les intersections}

Le premier résultat que nous montrons est le suivant :

Proposition 1. Soient $X$ une variété analytique complexe (lisse), $Y \subset X$ un sous-ensemble analytique fermé de codimension $q$, et $f: Y \longrightarrow \mathbb{C}$ une fonction holomorphe. Soit

$$
\Omega=\left\{\gamma \in \mathcal{C}_{q}(X) \mid \gamma \cap Y \text { est fini }\right\} .
$$

Alors l'application $\operatorname{Tr}_{Y} f: \Omega \longrightarrow \mathbb{C}$ donnée par

$$
\operatorname{Tr}_{Y} f(\gamma)=\sum_{x \in \gamma \cap Y} f(x)
$$

est holomorphe sur $\Omega$ et localement bornée sur $\bar{\Omega}$. En outre, $\bar{\Omega}$ est une réunion de composantes irréductibles de $\mathcal{C}_{q}(X)$.

Preuve. Dans ce paragraphe, $p_{1}$ (resp. $p_{2}$ ) est la restriction de la projection naturelle de $\mathcal{C}_{q}(X) \times X$ sur $\mathcal{C}_{q}(X)$ (resp. $X$ ) au "graphe" $G$ de la famille universelle de $q$-cycles paramétrée par $\mathcal{C}_{q}(X)$ dans $\mathcal{C}_{q}(X) \times X$.

Remarquons d'abord que si $\Gamma$ est une composante irréductible de $\mathcal{C}_{q}(X)$, alors $\Gamma \backslash \Omega$ est analytique dans $\Gamma$. En effet, si nous posons $G_{Y}=G \cap\left(\mathcal{C}_{q}(X) \times Y\right)$, alors

$$
D_{Y}=\left\{(\gamma, y) \in G_{Y} \mid \operatorname{dim}_{(\gamma, y)} p_{1 \mid G_{Y}}^{-1}(\gamma) \geq 1\right\}
$$

est analytique dans $G_{Y}$ par semi-continuité de la dimension des fibres. Comme $p_{1}$ est propre, $\Gamma \backslash \Omega=p_{1}\left(D_{Y}\right) \cap \Gamma$ est analytique dans $\Gamma$. Par conséquent, comme $\Omega$ est ouvert, $\bar{\Omega}$ est une réunion de composantes irréductibles de $\mathcal{C}_{q}(X)$.

Par ailleurs, d'après [Ba75], chapitre VI, théorème 10 (global), il existe une application holomorphe d'intersection $I: \Omega \longrightarrow \mathcal{C}_{0}(X)$ qui à $\gamma \in \Omega$ associe le cycle intersection avec $Y$. Ainsi, l'application $\operatorname{Tr}_{Y} f$, telle que nous l'avons définie ci-dessus, est holomorphe sur $\Omega$.

Montrons que $\operatorname{Tr}_{Y} f$ est localement bornée sur $\bar{\Omega}$ : soient $\gamma_{0} \in \bar{\Omega}$ (qui est comme on vient de le voir une union de composantes irréductibles de $\mathcal{C}_{q}(X)$ ), et $\mathcal{L}=\mathcal{L}_{1} \cup \cdots \cup \mathcal{L}_{r}$ un voisinage compact de $\gamma_{0}$ dans $\bar{\Omega}$, où chaque $\mathcal{L}_{i}$ est inclus dans une composante irréductible de $\bar{\Omega}$. Par propreté de la projection $p_{1}$, $L:=p_{2}\left(p_{1}^{-1}(\mathcal{L})\right)$ est un compact de $X$. Par ailleurs, pour $\gamma$ générique dans $\mathcal{L}_{i}$, $\gamma \cap Y$ est de cardinal $k_{i} \in \mathbb{N}^{\star}$ (en tenant compte des multiplicités). Soit $M$ une constante majorant $|f|$ sur le compact $L \cap Y$; alors $k M$ majore $\left|\operatorname{Tr}_{Y} f\right| \operatorname{sur} \Omega \cap L$ si $k=\max _{i=1, \ldots, r} k_{i}$.

Donnons maintenant une version améliorée de cet énoncé qui sera utile dans nos applications. 
Théorème 1. Dans la même situation que la proposition 1, il existe une application $F: \bar{\Omega} \longrightarrow \mathbb{C}$ holomorphe, telle que $F_{\mid \Omega}=\operatorname{Tr}_{Y} f$.

La preuve de ce théorème repose sur le

Lemme 1. Soient $X$ une variété analytique (lisse) et $Y \subset X$ un sous-ensemble analytique de codimension $q$. Soit $c_{Y} \in H_{Y}^{q}\left(X, \Omega_{X}^{q}\right)$ la classe fondamentale de $Y$ dans $X$.

Alors, pour tout $\gamma \in \mathcal{C}_{q}(X)$ tel que $|\gamma| \cap Y$ est fini, et pour toute $f \in \mathcal{O}(Y)$, on a

$$
\int_{\gamma} f c_{Y}=\operatorname{trace}_{\gamma \cap Y}(f)
$$

Preuve. Pour la construction de la classe fondamentale d'un $q$-cycle, nous renvoyons à [Ba80]. Précisons pour débuter le sens que l'on donne à $f c_{Y}$ : localement, près de $y \in Y, f$ est induite par une fonction holomorphe $\tilde{f}$ définie sur un voisinage $U$ de $y$ dans $X$. On peut donc définir un élément $\widetilde{f}\left(c_{Y}\right)_{\mid U} \in$ $H_{Y \cap U}^{q}\left(U, \Omega_{X}^{q}\right)$. Par ailleurs, si $\mathcal{I}_{Y} \subset \mathcal{O}_{X}$ est l'idéal réduit de $Y$, on a $\mathcal{I}_{Y} \cdot c_{Y}=0$ dans le faisceau $\underline{H}_{Y}^{q}$, et $H_{Y}^{q}\left(X, \Omega_{X}^{q}\right)=H^{0}\left(Y, \underline{H}_{Y}^{q}\left(\Omega_{X}^{q}\right)\right)$ (cf. [Ba80]). On peut donc globaliser la construction locale par recollement et obtenir un élément (unique) $f c_{Y} \in H_{Y}^{q}\left(X, \Omega_{X}^{q}\right)$. Ensuite, $\int_{\gamma} f c_{Y}$ est calculée en intégrant sur $\gamma$ l'image de $f c_{Y}$ dans $H^{q}\left(X, \Omega_{X}^{q}\right)$.

Il est bien connu que dans l'espace lisse $X$, on a les égalités suivantes :

$$
\int_{\gamma} f c_{Y}=\int_{X} f c_{\gamma} \smile c_{Y}=\int_{X} f c_{\gamma \cap Y}=\int_{\gamma \cap Y} f=\operatorname{trace}_{\gamma \cap Y}(f)
$$

où $c_{\gamma} \smile c_{Y} \in H_{c}^{N}\left(X, \Omega_{X}^{N}\right)$ désigne le cup-produit de la classe fondamentale de $\gamma$ avec celle de $Y$, et où $\gamma \cap Y$ est le cycle intersection de $\gamma$ et $Y$ (tenant compte des multiplicités), $c_{\gamma \cap Y}$ désignant sa classe fondamentale.

Preuve du théorème 1. La fonction

$$
\begin{aligned}
& \bar{\Omega} \longrightarrow \mathbb{C} \\
& \gamma \longmapsto \int_{\gamma} f c_{Y}
\end{aligned}
$$

coïncide avec $\operatorname{Tr}_{Y} f$ sur $\Omega$ d'après le lemme qui précède, et est holomorphe sur $\bar{\Omega}$ d'après [BV89].

Remarque. On peut s'affranchir de l'hypothèse de lissité de $X$ dans le théorème précédent à condition de supposer que $Y$ est une intersection complète locale (comparer avec [BM98]). 


\section{Applications}

\subsection{Convexité de l'espace des cycles des espaces $S$-holomorphiquement convexes.}

Nous débutons par un bref aperçu historique des résultats concernant la convexité holomorphe de l'espace des cycles. Elle a d'abord été étudiée par A. Andreotti et F. Norguet dans [AN67], où ils réinterprètent en particulier les résultats de [AN66] sur les $(q, q)$-classes de cohomologie. A la fin des années soixante, l'espace analytique des cycles compacts n'est pas encore construit dans le cas général, et ils se placent donc dans la situation d'une variété quasi-projective. Ils montrent que si $X$ est une variété quasi-projective lisse, "strictement" $q$-convexe, alors $\mathcal{C}_{q}(X)$ est holomorphiquement convexe, quitte à le normaliser faiblement. En 1977, F. Norguet et Y.-T. Siu améliorent ce résultat [NS77] en remplaçant l'hypothèse de $q$-convexité stricte par la notion plus classique de $q$-convexité forte ${ }^{1}$.

Parallèlement, en 1975 , le premier auteur montre que $\mathcal{C}_{q}(X)$ peut être muni d'une structure d'espace analytique de dimension finie dans le cas général [Ba75]. Puis, il montre que si $X$ est fortement $q$-convexe quelconque, il suffit que le lieu de dégénérescence de l'exhaustion possède un voisinage kählérien pour que $\mathcal{C}_{q}(X)$ soit holomorphiquement convexe [Ba78]. On retrouve ainsi, grosso modo, tous les résultats antérieurs et on constate en particulier l'importance de la kählériannité dans le cas quasi-projectif.

Plus précisément, si $\omega$ est une métrique hermitienne sur $X$, on peut définir le volume des éléments de $\mathcal{C}_{q}(X) \operatorname{par}_{\operatorname{vol}}(\gamma)=\int_{\gamma} \omega^{q}$. Or, d'après un théorème de Bishop, une partie $A$ de $\mathcal{C}_{q}(X)$ est relativement compacte si et seulement si l'on peut trouver un compact $K$ de $X$ et une constante $C$ tels que tout $\gamma$ de $A$ vérifie

(i) le support de $\gamma$ est inclus dans $K$;

(ii) $\operatorname{vol}_{\omega}(\gamma) \leq C$.

Mais, dans le cas kählérien, $\operatorname{vol}_{\omega}$ est localement constant $\operatorname{sur} \mathcal{C}_{q}(X)(\operatorname{car} \mathrm{d} \omega=$ $0)$. Par conséquent, dans ce cadre, une suite $\left(\gamma_{n}\right)$ d'une composante irréductible de l'espace des cycles admet une sous-suite discrète si et seulement si les supports des $\gamma_{n}$ ne sont inclus dans aucun compact de $X$, ce qui simplifie évidemment les considérations de compacité dans $\mathcal{C}_{q}(X)$.

Nous nous proposons maintenant d'étudier l'espace des q-cycles compacts d'un espace $S$-convexe, notion que nous allons définir.

Définition 1. Soit $X$ un espace analytique complexe muni d'un morphisme analytique $\pi: X \longrightarrow S$ où $S$ est analytique compact irréductible, de dimension q. On dit que $X$ est $S$-(holomorphiquement) convexe si, pour tout compact $K$

\footnotetext{
${ }^{1}$ Rappelons que nous avons adopté les conventions de [AN67] pour la $q$-convexité (0complet $=$ Stein)
} 
de $X$, l'ensemble

$$
\widehat{K}:=\left\{x \in X|| f(x)\left|\leq \sup _{y \in K_{\pi(x)}}\right| f(y) \mid, \forall f \in \mathcal{O}\left(X_{\pi(x)}\right)\right\}
$$

est relativement compact dans $X$.

Précisons que si $E$ est un sous-ensemble de $X$ et $s \in S$, nous notons $E_{s}$ l'intersection de $E$ avec $\pi^{-1}(s)$. Si $K$ est un compact de $X$, les ensembles $\widehat{K}_{s}$ sont tous fermés mais il n'y a pas de raison pour que $\widehat{K}$ le soit. Notre définition implique bien sûr que pour chaque $s \in S, X_{s}$ est holomorphiquement convexe. Les espaces $q$-holomorphiquement convexes (voir [BS93] et [Ko98]) sont des exemples d'espaces $S$-convexes, avec $S=\mathbb{P}_{q}$. Les espaces $q$-holomorphiquement convexes ne sont en général que faiblement q-convexes (car propres sur leur réduction qcomplète) donc nous ne pouvons pas utiliser directement les résultats de [Ba78].

La méthode d'intersection, grâce au théorème 1 , va cependant nous permettre de construire dans ce contexte "faiblement $q$-convexe" assez de fonctions holomorphes sur l'espace des $q$-cycles pour prouver le résultat d'holomorphe convexité suivant.

Théorème 2. Soient $X$ un espace $S$-holomorphiquement convexe kählérien irréductible et $\Gamma$ une composante irréductible de $\mathcal{C}_{q}(X)$. Supposons qu'un cycle $\gamma$ de $\Gamma$ a toutes ses composantes irréductibles qui se surjectent sur $S$. Alors, $\Gamma$ est holomorphiquement convexe.

Remarque 1. Ci-dessous, nous fournissons une autre preuve (plus courte) de ce théorème dans le cas un peu plus restrictif où $X$ est $q$-holomorphiquement convexe kählérien. Elle est bien moins "élémentaire".

Remarque 2. L'hypothèse de kählériannité de $X$ peut être affaiblie en demandant seulement l'existence sur $X$ d'une métrique hermitienne de classe $\mathcal{C}^{0}$ telle que la fonction continue $\operatorname{vol}_{\omega}: \Gamma \longrightarrow \mathbb{R}^{+}$vérifie, pour tout compact $K$ de $X$, $\sup _{|\gamma| \subset K}\left\{\operatorname{vol}_{\omega}(\gamma)\right\}<+\infty$.

Nous déduirons le théorème 2 de la proposition qui suit. $\operatorname{Si} \gamma \in \mathcal{C}_{q}(X)$, nous notons $\gamma^{h}$ ( $h$ pour horizontal) l'union des composantes irréductibles de $\gamma$ qui se surjectent sur $S$. Comme $S$ est compact et irréductible de dimension $q$, cela signifie qu'une telle composante irréductible "horizontale" est génériquement finie sur $S$.

Proposition 2. Soient $X$ un espace S-holomorphiquement convexe irréductible et $\Gamma$ une composante irréductible de $\mathcal{C}_{q}(X)$. Supposons qu'une suite $\left(\gamma_{n}\right)$ est discrète dans $\Gamma$ si et seulement si aucune sous-suite de $\left(\left|\gamma_{n}^{h}\right|\right)$ n'est incluse dans un compact de $X$. Alors, $\Gamma$ est holomorphiquement convexe.

Preuve. Ici, $p_{1}$ (resp. $p_{2}$ ) est la restriction de la projection naturelle de $\Gamma \times X$ sur $\Gamma($ resp. $X)$ au graphe $G$ de la famille de $q$-cycles paramétrée par $\Gamma$ dans $\Gamma \times X$. 
Nous pouvons supposer que $\Gamma$ n'est pas compacte (sinon la proposition est triviale). Dans ce cas, l'hypothèse montre qu'il existe $\gamma$ tel que $\gamma^{h} \neq \emptyset$. Remarquons alors que tous les cycles de $\Gamma$ s'envoient surjectivement sur $S$. D'une part, pour que $\gamma$ se surjecte sur $S$, il faut et il suffit que $\pi_{\mid \gamma}$ soit ouvert en un point de $\gamma(\operatorname{car} \pi(\gamma)$ est analytique dans $S)$, et cette condition est ouverte sur $\Gamma$. D'autre part, s'il existe un cycle $\gamma_{0}$ dont l'image par $\pi$ n'est pas $S$, prenons un voisinage $V$ (distinct de $S$ ) de l'ensemble analytique $\pi\left(\gamma_{0}\right)$ : il existe un voisinage $U$ de $\gamma_{0}$ dans $\Gamma$ tel que pour tout $\gamma \in U, \pi(\gamma)$ est contenu dans $V$, ce qui signifie que l'ensemble des $\gamma$ de $\Gamma$ qui se surjectent est fermé. Enfin, cet ensemble n'est pas vide.

Notons maintenant $\mathcal{M}$ l'ensemble des points de $S$ tels que $\pi^{-1}(s)$ n'est pas "intersection complète locale," ou pas de codimension pure $q$. C'est un sousensemble maigre $^{2}$ de $S$. En effet, $S$ est lisse en tout point $s$ d'un ouvert de Zariski dense. Or, pour un tel $s, \pi^{-1}(s)$ est intersection complète locale si et seulement si $\pi$ est plat le long de cette fibre (qui est dans ce cas de codimension pure $q$ car $X$ est irréductible). Mais, d'après un résultat de J. Frisch [Fr67], l'ensemble des points de non platitude de $\pi$ est d'image maigre dans $S$.

Fixons alors $\gamma_{0} \in \Gamma$. L'ensemble $\mathcal{M}_{\gamma_{0}}$ des points de $S$ qui, soit sont dans $\mathcal{M}$, soit sont tels que $\gamma_{0_{s}}$ n'est pas finie, est maigre dans $S$ par les deux remarques que nous venons de faire. Pour $s \in S \backslash \mathcal{M}_{\gamma_{0}}$, notons $\Gamma_{s}$ l'ensemble des $\gamma \in \Gamma$ tels que $\gamma_{s}$ est finie : c'est un ouvert de Zariski (non vide) de $\Gamma$ d'après le théorème 1. En outre, pour toute $f \in \mathcal{O}\left(\pi^{-1}(s)\right)$, nous avons une fonction holomorphe

$$
\operatorname{Tr}_{s} f: \Gamma_{s} \longrightarrow \mathbb{C}
$$

qui est l'application de trace de $f$ sur la fibre $\gamma_{s}$. En posant $Y=X_{s}$, la fonction $\operatorname{Tr}_{Y} f$ du théorème 1 coïncide avec $\operatorname{Tr}_{s} f$ sur $\Gamma_{s}$ et est holomorphe sur $\Gamma$ (voir la remarque qui suit la preuve du théorème 1). Dans ce qui suit, nous identifions $\operatorname{Tr}_{X_{s}} f$ et $\operatorname{Tr}_{s} f$.

Pour montrer que $\Gamma$ est holomorphiquement convexe, prenons $\mathcal{K}$ un compact de $\Gamma$ et considérons $K:=p_{2}\left(p_{1}^{-1}(\mathcal{K})\right)$; c'est un compact de $X$ ( $p_{1}$ est propre). Choisissons en outre $\mathcal{K}$ de sorte que $\mathcal{K}=\mathcal{K}$. L'ensemble $\widehat{K}$ (au sens $S$-convexe) est par hypothèse relativement compact. Donc, si $\Gamma_{\widehat{K}} \subset \Gamma$ est l'ensemble des cycles dont les composantes "horizontales" sont contenues dans la fermeture de $\widehat{K}$, il suffit de montrer que $\widehat{\mathcal{K}} \subset \Gamma_{\widehat{K}}$, où cette fois $\widehat{\mathcal{K}}$ désigne l'enveloppe holomorphiquement convexe classique de $\mathcal{K}$ dans $\Gamma$.

Mais, si $\left|\gamma_{0}^{h}\right| \not \subset \widehat{K}$, il existe une composante irréductible $\gamma_{0}^{\prime}$ de $\gamma_{0}$ génériquement finie sur $S$ et non incluse dans la fermeture de $\widehat{K}$. Ainsi, on peut trouver un point $x \in \gamma_{0}^{\prime} \backslash \widehat{\widehat{K}}$ tel que $s=\pi(x)$ n'appartient pas à $\mathcal{M}_{\gamma_{0}}$ (son complémentaire est dense dans $S$ ), et une fonction holomorphe sur $X_{s}$ telle que

\footnotetext{
${ }^{2}$ C'est-à-dire localement réunion dénombrable de sous-ensembles analytiques localement fermés d'intérieur vide
} 
$|f(x)|>1>1 / 2 k>\sup _{y \in \widehat{K}_{s}}|f(y)|$, où $k$ est le degré générique des cycles de $\Gamma$ sur $S$.

Il nous suffit par conséquent de trouver un entier $\lambda>0$ tel que

$$
\left|\operatorname{Tr}_{s} f^{\lambda}\left(\gamma_{0}\right)\right|>1
$$

car nous avons déjà $\left|\operatorname{Tr}_{s} f^{\lambda}(\gamma)\right|<1 / 2$ quel que soit $\gamma \in \Gamma_{s}$ contenu dans $\widehat{K}$; en effet, nous obtenons le résultat annoncé puisque $\operatorname{Tr}_{s} f$ est holomorphe sur $\Gamma$, et la fermeture de $\mathcal{K} \cap \Gamma_{s}$ dans $\Gamma$ est égale à $\mathcal{K}\left(\Gamma_{s}\right.$ est un ouvert de Zariski (dense) de $\Gamma$ et $\stackrel{\overline{\mathcal{K}}}{=}=\mathcal{K}$ ).

Pour prouver l'inégalité $(*)$, on remarque que si $z_{1}, \ldots, z_{k}$ sont des nombres complexes situés dans un même quart de plan fermé, de sommet l'origine, alors

$$
\left|\sum_{j=1}^{k} z_{j}\right| \geq \max _{j=1, \ldots k}\left|z_{j}\right| \text {. }
$$

On est donc ramené à trouver, pour un $k$-uplet $z_{1}, \ldots z_{k}$ de nombres complexes quelconques fixés, un entier $\lambda$ strictement positif de sorte que $z_{1}^{\lambda}, \ldots, z_{k}^{\lambda}$ se trouvent dans le même quart de plan de sommet l'origine. En raisonnant sur les arguments de ces nombres complexes, le lemme classique suivant (de Dirichlet) répond à notre problème.

Lemme 2. Soient $\theta_{1}, \ldots, \theta_{N}$ des nombres réels positifs et $D$ un entier strictement positif. Pour tout $A \geq 2$, il existe un entier $\alpha, D \leq \alpha \leq D \cdot A^{N}$, tel que

$$
\max _{1 \leq j \leq N}\left(\min _{i \in \mathbb{N}}\left|\alpha \theta_{j}-i\right|\right) \leq 1 / A
$$

Preuve du lemme. L'ensemble $\left[0 ; 1\left[{ }^{N}\right.\right.$ est la réunion disjointe des $A^{N}$ ensembles

$$
C_{m_{1}, \ldots, m_{N}}:=\prod_{l=1}^{N}\left[\frac{m_{l}}{A} ; \frac{m_{l}+1}{A}\right], \quad 0 \leq m_{1}, \ldots, m_{N}<A .
$$

Si frac $\theta$ désigne la partie fractionnaire du réel positif $\theta$, parmi les $A^{N}+1 N$ uplets $\left(p D\right.$ frac $\theta_{1}, \ldots, p D$ frac $\left.\theta_{N}\right)\left(0 \leq p \leq A^{N}\right)$, deux au moins sont dans un même $C_{m_{1}, \ldots, m_{N}}$. En désignant par $p$ et $p^{\prime}$ les deux entiers correspondants, nous avons prouvé le lemme avec $\alpha=\left|p^{\prime}-p\right| D$.

Pour déduire le théorème 2 de la proposition 2, nous utiliserons le

Lemme 3. Soient $X$ un espace $S$-convexe et $\Gamma$ une composante irréductible de $\mathcal{C}_{q}(X)$. Supposons que pour $\gamma$ générique dans $\Gamma, \pi_{\mid \gamma}: \gamma \longrightarrow S$ est génériquement fini. Si un cycle $\gamma$ de $\Gamma$ est tel que $\left|\gamma^{h}\right| \subset K$, où $K$ est compact, alors il vérifie $|\gamma| \subset \overline{\widehat{K}}$ (ce dernier est aussi compact).

Preuve. Fixons un compact $K$ de $X$ et posons $L=\overline{\widehat{K}}$. Soit un cycle $\gamma_{0}$ de $\Gamma$ qui n'est pas inclus dans $L$, et soit un point $x \in\left|\gamma_{0}\right| \backslash L$. Nous notons $s_{0}=\pi(x)$, 
et $C_{0}, \ldots, C_{r}$ les différentes composantes connexes de $\gamma_{s_{s_{0}}}\left(x \in C_{0}\right)$. Comme $X$ est $S$-holomorphiquement convexe, $C_{0}$ est disjointe de $K$, car les fonctions holomorphes sont constantes sur $C_{0}$.

Soient $U_{0}, \ldots, U_{r}$ des voisinages respectifs de $C_{0}, \ldots, C_{r}$ dans $X$, deux à deux disjoints, et $U$ l'union de ces voisinages. On choisit $U_{0}$ de sorte qu'il ne rencontre pas $K$. Par propreté de $\pi_{\mid \gamma_{0}}$, il existe un voisinage $V$ de $s_{0}$ dans $S$ tel que $\pi^{-1}(\bar{V}) \cap\left|\gamma_{0}\right| \subset U$.

Par définition de la topologie dans $\Gamma$, il existe un voisinage de $\gamma_{0}$ dans $\Gamma$ tel que tout $\gamma$ dans ce voisinage vérifie $\pi^{-1}(\bar{V}) \cap|\gamma| \subset U$. Ainsi, pour chaque $\gamma$ assez proche de $\gamma_{0}, \pi_{\mid \gamma \cap \pi^{-1}(V) \cap U_{0}}$, à valeurs dans $V$, est propre. Par conséquent, pour tout $\gamma$ générique proche de $\gamma_{0}, \pi\left(|\gamma| \cap \pi^{-1}(V) \cap U_{0}\right)=V$, et donc cette égalité est vraie pour $\gamma_{0}$.

Finalement, nous déduisons que $\left|\gamma_{0}^{h}\right| \cap U_{0} \neq \emptyset$ et donc que $\left|\gamma_{0}^{h}\right| \not \subset K$.

Preuve du théorème 2. D'après le début de la démonstration de la proposition 2 , tous les cycles de $\Gamma$ se surjectent sur $S$, puisque l'un au moins se surjecte. Nous allons voir que notre hypothèse implique même que si $\gamma$ est un cycle générique de $\Gamma$, alors $\pi_{\mid \gamma}: \gamma \longrightarrow S$ est génériquement fini. Dès lors, le théorème 2 sera une conséquence immédiate de la proposition 2 et du lemme 3 .

Nous montrons donc dans ce qui suit que le cycle générique de $\Gamma$ est génériquement fini sur $S$. Soit $|G| \subset \Gamma \times X$ le graphe de la famille de cycles paramétrée par $\Gamma$. Posons $|G|=\cup_{i=1}^{k} G_{i}$ la décomposition en composantes irréductibles de $|G|$. Quitte à passer au normalisé $\widetilde{\Gamma}$ de $\Gamma$, on peut supposer que $\widetilde{G}_{i}:=\widetilde{\Gamma} \times_{\Gamma} G_{i}$ est le graphe d'une famille analytique de cycles paramétrée par $\widetilde{\Gamma}$ et génériquement sans multiplicité. On a alors des entiers $n_{1}, \ldots, n_{k} \in \mathbb{N}^{\star}$ tels que pour tout $\gamma \in \widetilde{\Gamma}$, on ait

$$
\gamma=\sum_{i=1}^{k} n_{i} \gamma_{i}
$$

où $\gamma_{i}$ est le cycle de la "famille" $\widetilde{G}_{i}$ associé à $\gamma \in \widetilde{\Gamma}$. Alors, pour tout $\gamma \in \widetilde{\Gamma}$, on a :

- $\left|\gamma_{i}\right|$ est réunion (non vide) de composantes irréductibles de $|\gamma|$;

- si $\gamma \in \widetilde{\Gamma}$ est générique, $\gamma_{i}=\left|\gamma_{i}\right|$ (et donc $\gamma=\sum_{i=1}^{k} n_{i}\left|\gamma_{i}\right|$ ).

En fait, la famille initiale "relevée" à $\widetilde{\Gamma}$ est donnée par le cycle $\sum_{i=1}^{k} n_{i}\left|\widetilde{G}_{i}\right|$ de $\widetilde{\Gamma} \times X$ (c'est la pondération géométriquement plate associée au morphisme $|\widetilde{G}| \longrightarrow \widetilde{\Gamma}$ donnant le pull-back sur $\widetilde{\Gamma}$ de la famille initiale, voir [BM98]).

Maintenant, si on a un ouvert non vide $\Omega$ de $\Gamma$ tel que pour tout $\gamma \in \Omega$, une composante irréductible de $|\gamma|$ ne se surjecte pas sur $S$, alors il existe $i \in[1 ; k]$ avec un ouvert non vide ${ }^{3} \widetilde{\Omega}$ de $\widetilde{\Gamma}$ tel que pour $\gamma \in \widetilde{\Omega}, \gamma_{i}$ a une composante irréductible ne se surjectant pas sur $S$. On a donc un ouvert non vide de $\widetilde{G}_{i}$

\footnotetext{
${ }^{3}$ Sinon, pour chaque $i$, les $\gamma$ ayant une composante de $\gamma_{i}$ qui ne se surjecte pas est un fermé d'intérieur vide (de Zariski) et leur réunion pour $i=1, \ldots, k$ ne peut contenir $\Omega$
} 
sur lequel le rang de $\tau_{i}: \widetilde{G}_{i} \longrightarrow \widetilde{\Gamma} \times S$ n'est pas maximum. Comme $\operatorname{dim} \widetilde{G}_{i}=$ $\operatorname{dim}(\widetilde{\Gamma} \times S)$, et $\widetilde{G}_{i}$ est irréductible, l'image de $\tau_{i}$ est d'intérieur vide dans $\widetilde{\Gamma} \times S$.

Mais, si $\gamma^{0} \in \Gamma$ est le cycle dont toutes les composantes irréductibles se surjectent sur $S,\left|\gamma_{i}^{0}\right|$ se surjecte sur $\left\{\gamma^{0}\right\} \times S$ (et même chacune de ses composantes irréductibles) et il en sera donc de même pour $\gamma$ voisin de $\gamma_{0}$. Alors, l'image de $\tau_{i}$ contient un ouvert, d'où une contradiction.

Par conséquent, pour $\gamma$ générique, toute composante irréductible de $|\gamma|$ se surjecte sur $S$ (et est donc génériquement finie sur $S$ ).

Voici une autre preuve du théorème 2 dans le cas $q$-holomorphiquement convexe (au sens de [BS93]), en utilisant un résultat de [Ba78]. On dispose dans ce cas d'un morphisme (propre) de réduction $\rho: X \longrightarrow \widetilde{X}$ où $\widetilde{X}$ est $q$-Stein, et d'un diagramme

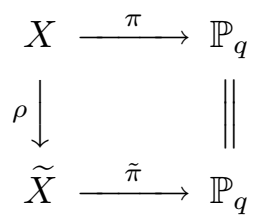

commutatif (voir [BS93]).

Il existe alors, d'après [Ba75], un morphisme d'image directe $\rho_{\star}: \Gamma \longrightarrow \mathcal{C}_{q}(\widetilde{X})$ qui est holomorphe ${ }^{4}$ et qui consiste à oublier les composantes irréductibles de l'image d'un cycle qui ne sont pas de dimension $q$. Ce morphisme est propre car $\rho$ l'est et $X$ est kählérien. Comme $\widetilde{X}$ est $q$-Stein, il est $q$-complet, ce qui implique que $\mathcal{C}_{q}(\widetilde{X})$ est de Stein ([Ba78]). Notre théorème en découle dans ce cas; on remarquera que bien que courte, cette preuve fait appel à des résultats profonds.

\subsection{Espace des cycles et $q$-convexité locale.}

Soit $U \subset \subset X$ un ouvert relativement compact à bord $\mathcal{C}^{2}$ dans une variété complexe lisse $X$ de dimension $N$. Soient $q \in \mathbb{N}$ et $\gamma_{0}$ un $q$-cycle de $X$ (compact) vérifiant $\left|\gamma_{0}\right| \subset \bar{U}$ et $\left|\gamma_{0}\right| \not \subset U$. Le cycle $\gamma_{0}$ est donc dans le bord de l'ouvert $\mathcal{C}_{q}(U)$ de $\mathcal{C}_{q}(X)$. Soit $x_{0} \in \partial U \cap\left|\gamma_{0}\right|$ et supposons que $U$ est fortement $q$-convexe en $x_{0}$. Dans une carte de $X$ autour de $x_{0}$, on peut définir (localement) $U$ par $U=\{\rho<0\}$ où $\rho$ est de classe $\mathcal{C}^{2}$ au voisinage de $x_{0}$. On peut aussi choisir des coordonnées $z_{1}, \ldots, z_{N}$, centrées en $x_{0}$, telles que l'on ait

$$
\rho(z)=\operatorname{Re}\left(z_{N}\right)+\sum_{j=q+1}^{N}\left|z_{j}\right|^{2}+\sum_{k=1}^{q} a_{k}\left|z_{k}\right|^{2}+o\left(\|z\|^{2}\right),
$$

où les $a_{j}$ sont dans $\mathbb{R}$ (éventuellement négatifs).

Remarquons alors que si $Y_{0}=\left\{z_{1}=\cdots=z_{q}=0\right\}$, la fonction $\rho$ est strictement plurisousharmonique sur $Y_{0}$ près de $x_{0}$. On peut donc trouver un $(N-q)$ plan (linéaire dans ces coordonnées) passant par $x_{0}$, noté $Y$, tel que $\rho_{\mid Y}$ soit

\footnotetext{
${ }^{4}$ Ce résultat n'est pas dans [Ba75] mais la preuve du théorème d'image directe (voir le chapitre IV), s'adapte "telle quelle" à ce cas plus général
} 
encore strictement psh près de $x_{0}$ et tel que $x_{0}$ soit un point isolé de $\left|\gamma_{0}\right| \cap Y$; notons $d \in \mathbb{N}^{\star}$ la multiplicité de $x_{0}$ dans cette intersection.

On construit alors facilement une $q$-écaille $E$ adaptée à $\gamma_{0}$ au voisinage de $x_{0}$, de la forme $\Delta_{q} \times Y^{\prime}$, où $Y^{\prime}$ est un polydisque de centre $x_{0}$ dans $Y$ assez petit, et où $\Delta_{q}$ est un polydisque de $\mathbb{C}^{q}$ (du type $\left|z_{i}\right|<\varepsilon$ pour $i \in[1 ; q]$ et $z_{j}=0$ pour $j \geq q+1$ ), de sorte que

(i) $\left(\bar{\Delta}_{q} \times \partial Y^{\prime}\right) \cap\left|\gamma_{0}\right|=\emptyset \quad\left(E\right.$ adaptée à $\left.\gamma_{0}\right)$;

(ii) $\operatorname{deg}_{E} \gamma_{0}=d$ (i.e. $\left.\gamma_{0} \cap\left(\{0\} \times Y^{\prime}\right)=d\left\{x_{0}\right\}\right)$.

Notons $\Omega(E)$ l'ouvert de $\mathcal{C}_{q}(X)$ défini par le conditions (i) et (ii) et posons

$$
\mathcal{V}=\left\{\gamma \in \Omega(E)|| \gamma \mid \cap\left(\{0\} \times Y^{\prime}\right) \cap \partial U=\emptyset \text { et }|\gamma| \cap\left(\{0\} \times Y^{\prime}\right) \subset U\right\}
$$

Remarquons que $\mathcal{C}_{q}(U) \cap \Omega(E) \subset \mathcal{V}$.

Pour $f \in \mathcal{O}\left(Y^{\prime} \cap U\right)$, on a alors une fonction holomorphe

$$
\begin{aligned}
F: \mathcal{V} & \longrightarrow \mathbb{C} \\
\gamma & \longmapsto \operatorname{trace}_{\gamma \cap\left(\{0\} \times Y^{\prime}\right)}(f) .
\end{aligned}
$$

Comme $Y^{\prime} \cap U$ est de Stein (car défini dans $Y^{\prime}$ de Stein par $\{\rho<0\}$ avec $\rho$ strictement psh), si on a $\left(\gamma_{n}\right)$ une suite de $\mathcal{C}_{q}(U)$ vérifiant $\lim _{n \rightarrow+\infty} \gamma_{n}=\gamma_{0}$ dans $\mathcal{C}_{q}(X)$, on obtiendra aisément une fonction holomorphe $F$ sur $\mathcal{C}_{q}(U) \cap \Omega(E)$ vérifiant

$$
\limsup _{n \rightarrow+\infty}\left|F\left(\gamma_{n}\right)\right|=+\infty
$$

(voir par exemple la proposition 3 ci-après).

Nous allons constater que ce phénomène peut être étendu au voisinage de $\gamma_{0}$ si l'on impose de la $q$-convexité forte en tout point de $\left|\gamma_{0}\right| \cap \partial U$.

Théorème 3. Soit $U \subset \subset X$ et soit $\gamma_{0} \in \partial \mathcal{C}_{q}(U)$. Si $U$ est fortement $q$-convexe en tout point de $\left|\gamma_{0}\right| \cap \partial U$, alors $\mathcal{C}_{q}(U)$ est localement Stein en $\gamma_{0}$ (i.e. il existe un voisinage de $\gamma_{0}$ dans $\mathcal{C}_{q}(X)$ dont l'intersection avec $\mathcal{C}_{q}(U)$ est Stein).

Preuve. Recouvrons $\left|\gamma_{0}\right| \cap \partial U$ par un nombre fini d'écailles $E_{1}, \ldots, E_{p}$ adaptées à $\gamma_{0}$. Notons $\Omega^{\prime}=\Omega\left(E_{1}\right) \cap \cdots \cap \Omega\left(E_{p}\right)$ et choisissons un voisinage ouvert relativement compact de Stein $\Omega^{\prime \prime}$ de $\gamma_{0}$ dans $\mathcal{C}_{q}(X)$ vérifiant

(1) $\Omega^{\prime \prime} \subset \subset \Omega^{\prime}$

(2) pour tout $\gamma \in \overline{\Omega^{\prime \prime}} \cap \partial \mathcal{C}_{q}(U)$, on a $|\gamma| \cap \partial U \subset \bigcup_{j=1}^{p} E_{j}$.

Montrons qu'alors $\Omega^{\prime \prime} \cap \mathcal{C}_{q}(U)$ est de Stein. Il suffit pour cela de prouver la convexité holomorphe (car $\Omega^{\prime \prime}$ est Stein); pour chaque suite discrète $\left(\gamma_{n}\right)_{n \in \mathbb{N}}$ dans $\Omega^{\prime \prime} \cap \mathcal{C}_{q}(U)$, nous sommes donc amenés à trouver $F$ holomorphe sur $\Omega^{\prime \prime} \cap$ $\mathcal{C}_{q}(U)$ vérifiant

$$
\limsup _{n \rightarrow+\infty}\left|F\left(\gamma_{n}\right)\right|=+\infty
$$


On peut passer à une sous-suite et par conséquent supposer que

$$
\lim _{n \rightarrow+\infty} \gamma_{n}=\gamma \in \mathcal{C}_{q}(X)
$$

Si $\gamma \in \partial \Omega^{\prime \prime}$, le résultat est facile car on peut déjà trouver $F \in \mathcal{O}\left(\Omega^{\prime \prime}\right)$ vérifiant $(* *)$. Sinon, $\gamma \in \partial \mathcal{C}_{q}(U)$ et il existe $x \in|\gamma| \cap \partial U$. D'après la condition (2), il existe $j \in[1 ; p]$ avec $x \in E_{j}$. Soit $\delta$ tel que $x \in\{\delta\} \times Y_{j}^{\prime}$. Alors, les intersections $\gamma_{n} \cap\left(\{\delta\} \times Y_{j}^{\prime}\right)$ convergent vers $\gamma \cap\left(\{\delta\} \times Y_{j}^{\prime}\right)$ dans $\mathcal{C}_{0}\left(\{\delta\} \times Y_{j}^{\prime}\right)$ (d'après $(1)$, on a $\gamma_{n} \in \Omega\left(E_{j}\right)$, donc ces intersections sont des $\left(d_{j}\right)$-uplets de $\left.\left(\{\delta\} \times Y_{j}^{\prime}\right) \cap U\right)$.

D'après ce qui a été vu plus haut, on trouve alors $f \in \mathcal{O}\left(\left(\{\delta\} \times Y_{j}^{\prime}\right) \cap U\right)$ de sorte que la fonction $F$ sur $\Omega^{\prime \prime} \cap \mathcal{C}_{q}(U)$ définie par

$$
F(\widetilde{\gamma})=\operatorname{Tr}_{\{\delta\} \times Y_{j}^{\prime}} f(\widetilde{\gamma})
$$

soit holomorphe et vérifie $\lim _{n \rightarrow+\infty}\left|F\left(\gamma_{n}\right)\right|=+\infty$.

Une éventuelle globalisation de ce résultat aboutirait à un théorème de convexité holomorphe de $\mathcal{C}_{q}(U)$; mais on ne peut espérer plus que les résultats de [Ba78] et l'amélioration donnée dans [Ba87]; aussi ne poursuivons nous pas dans cette direction, le point nouveau étant précisément l'aspect local du "transfert de convexité".

\subsection{Fonctions holomorphes sur $\mathcal{C}_{q}(X)$ pour $X$ ouvert d'une variété} projective.

La proposition qui suit est une conséquence simple du théorème 1; elle permet à J.A. Wolf [Wo00] de montrer que certaines composantes de l'espace des cycles sont Stein, dans le cas de certains domaines des variétés drapeaux.

Proposition 3. Soient $Z$ une variété analytique complexe kählérienne, $E \subset Z$ un fermé, et posons $X=Z \backslash E$. Fixons $q \in \mathbb{N}^{\star}$, et $\Gamma$ une composante irréductible de $\mathcal{C}_{q}(X)$. Supposons que $\Gamma$ est relativement compacte dans $\mathcal{C}_{q}(Z)$ et que, pour tout $\gamma \in \bar{\Gamma} \backslash \Gamma$, il existe un sous-ensemble analytique $Y_{0} \subset Z$ de codimension $q$ tel que :

$-\gamma \cap Y_{0} \cap E \neq \emptyset$;

- $Y=Y_{0} \backslash E$ est Stein, alors $\Gamma$ est holomorphiquement convexe.

Si en outre, pour tous $\gamma_{1}$ et $\gamma_{2}$ distincts dans $\Gamma$, il existe $Y_{0}$ de codimension $q$ qui intersecte $\gamma_{1}$ et $\gamma_{2}$ de façon distincte, et tel que $Y$ soit Stein, alors $\Gamma$ est Stein.

Preuve. Avec la première hypothèse, nous montrons que pour toute suite discrète $\left(\gamma_{n}\right)$ d'éléments de $\Gamma$, il existe $F \in \mathcal{O}(\Gamma)$ non bornée sur $\left(\gamma_{n}\right)$.

Il existe $\gamma \in \mathcal{C}_{q}(Z)$ qui est valeur d'adhérence de la suite $\left(\gamma_{n}\right)$, et $Y \subset X$ de Stein, de codimension $q$, tels que $\bar{Y} \cap|\gamma| \cap E \neq \emptyset$. Le sous-ensemble analytique $Y$ rencontre donc tous les éléments de $\Gamma$ et, pour tout $n$, l'ensemble $\left|\gamma_{n}\right| \cap Y$ est 
fini (de cardinal $d$ ) car $Y$ est Stein. Quitte à prendre une sous-suite, on peut supposer que $\left(\gamma_{n}\right)$ a pour limite $\gamma$ et que $\left(\gamma_{n} \cap Y\right) \in \operatorname{Sym}^{d}(X)$ a une limite dans $\operatorname{Sym}^{d}(Z)$. En utilisant le fait que $\left(\gamma_{n}\right)$ est discrète, et $\bar{Y} \cap|\gamma| \cap E \neq \emptyset$, on peut écrire,

$$
\bigcup_{n \in \mathbb{N}}\left\{\left|\gamma_{n}\right| \cap Y\right\}=\left\{x_{k}\right\}_{k \in \mathbb{N}} \cup\left\{y_{k}\right\}_{k \in \mathbb{N}}
$$

où $\left(x_{k}\right)$ est discrète dans $Y$, et $\left(y_{k}\right)$ (éventuellement finie) est incluse dans un compact de $Y$. Comme $Y$ est Stein, il existe une fonction holomorphe $f$ sur $Y$ telle que $f\left(x_{k}\right)=k$ pour tout $k$. Nous appliquons maintenant le théorème 1 à $Y$ et à $f$ que nous venons de choisir pour obtenir la fonction $F$ désirée.

Avec la seconde hypothèse, pour séparer deux points $\gamma_{1}$ et $\gamma_{2}$ distincts dans $\Gamma$, le procédé est encore plus simple : on choisit $Y$ qui rencontre $\gamma_{1}$ et $\gamma_{2}$ de façon distincte (et finie). Si $x_{1} \in\left(\left|\gamma_{1}\right| \cap Y\right) \backslash\left|\gamma_{2}\right|$ et $x_{2} \in\left(\left|\gamma_{2}\right| \cap Y\right) \backslash\left|\gamma_{1}\right|$, on choisit $f \in \mathcal{O}(Y)$ telle que $f\left(x_{1}\right)=0, f\left(x_{2}\right)=1, f$ nulle en tous les autres points d'intersection de $\gamma_{1}$ ou $\gamma_{2}$ avec $Y$. On applique ensuite le théorème 1 comme précédemment.

Nous considérons maintenant le cas suivant : $Z$ est une variété projective (lisse) connexe de dimension $N$, et $E \subset Z$ un sous-ensemble fermé non vide. Nous nous intéressons à $\mathcal{C}_{N-1}(X)$.

Corollaire. Dans la situation que nous venons de décrire, toute composante irréductible de $\mathcal{C}_{N-1}(X)$ est Stein.

Preuve. Soit $\Gamma$ une composante irréductible de $\mathcal{C}_{N-1}(X)$. Les conditions de la proposition 3 sont facilement vérifiées.

En effet, dans cette situation particulière, la compacité relative de $\Gamma$ dans $\mathcal{C}_{N-1}(Z)$ est bien connue. Par ailleurs, on dispose d'assez de courbes pour que les autres hypothèses soient vérifiées (une surface de Riemann irréductible non compacte étant de Stein).

Le lecteur remarquera que ce dernier résultat s'obtient aussi par une utilisation de [Ba78]. En effet, $X$ étant de dimension $N$, sans composante connexe compacte, il est $(N-1)$-complet (avec notre convention) d'après un théorème de T. Ohsawa [Oh84], ce qui implique que $\mathcal{C}_{N-1}(X)$ est de Stein. Une fois encore, cette argumentation courte passe par l'application de théorèmes difficiles.

Remarque. La proposition 3 peut aussi s'appliquer si $Z$ est projective et $E$ est un $(N-q-1)$-cycle compact. Toute composante irréductible $\Gamma$ de $\mathcal{C}_{q}(X)$ est alors relativement compacte dans $\mathcal{C}_{q}(Z)$. Pour avoir la convexité holomorphe de $\Gamma$, il "suffit" de trouver parmi tous les $(N-q)$-cycles compacts de $Z$ qui contiennent $E$, un $Y_{0}$ qui croise un élément de $\Gamma$ et tel que $Y_{0} \backslash E$ est Stein (par exemple, $E$ est un diviseur ample de $Y_{0}$ ). 


\section{Références}

[AN66] A. Andreotti and F. Norguet, Problème de Levi et convexité holomorphe pour les classes de cohomologie, Ann. Scuola sup. Pisa, 20 (1966), 197-242.

[AN67]__ Convexité holomorphe dans l'espace des cycles d'une variété algébrique, Ann. Scuola sup. Pisa, 21 (1967), 31-82.

[Ba75] D. Barlet, Espace analytique réduit des cycles analytiques complexes compacts d'un espace analytique complexe de dimension finie, Séminaire F. Norguet, Lecture Notes in Math., Springer Verlag 482 (1975), 1-158.

[Ba78] _ Convexité de l'espace des cycles, Bull. Soc. math. France 106 (1978), 373-397.

[Ba80] _ Familles analytiques de cycles et classes fondamentales relatives, Séminaire F. Norguet, Lecture Notes in Math., Springer-Verlag 807 (1980), 1-24.

[Ba87] , À propos d'une conjecture de R. Hartshorne, J. Reine Angew. Math. 374 (1987), 214-220.

[BM98] D. Barlet and J. Magnusson, Intégration de classes de cohomologie méromorphes et diviseurs d'incidence, Ann. Sc. Ec. Norm. Sup. 31 (1998), 811-842.

[BS93] D. Barlet and A. Silva, Convexité holomorphe intermédiaire, Math. Ann. 296 (1993), 649-665.

[BV89] D. Barlet and J. Varouchas, Fonctions holomorphes sur l'espace des cycles, Bull. Soc. Math. France 117 (1989), 327-341.

[Fr67] J. Frisch, Points de platitude d'un morphisme d'espaces analytiques complexes, Invent. Math. 4 (1967), 118-138.

[Ko98] V. Koziarz, Plongement des espaces q-Stein, Thèse de l'Université de Nancy-I (1998).

[NS77] F. Norguet, Y.-T. Siu, Holomorphic convexity of spaces of cycles, Bull. Soc. Math. France 105 (1977), 191-223.

[Oh84] T. Ohsawa, Completeness of noncompact analytic spaces, Publ. R.I.M.S., Kyoto Univ., 20 (1984), 683-692.

[Wo00] J. A. Wolf, Hermitian symmetric spaces, cycles spaces, and the Barlet-Koziarz method, Math. Res. Lett. 7 (2000), 551-564.

Université Henri-Poincaré (NAnCy-I) et Institut Universitaire de France, Institut Élie Cartan, U.M.R. 7502, B.P. 239, 54506 Vandoeuvre-Lès-Nancy Cedex, France.

E-mail address: barlet@iecn.u-nancy.fr

Université de Grenoble-I, Institut Fourier, Laboratoire de Mathématiques, U.M.R. 5582, B.P. 74, 38402 Saint-Martin D'Hères, France.

E-mail address: koziarz@iecn.u-nancy.fr 